\title{
ON $\delta$-SUPERDERIVATIONS OF SIMPLE SUPERALGEBRAS OF JORDAN BRACKETS
}

\author{
V. N. ZHELYABIN AND I. B. KAYGORODOV
}

\begin{abstract}
A complete description of $\delta$-derivations and $\delta$-superderivations is given for simple unital superalgebras of Jordan brackets over a field of characteristic different from 2 and for simple unital finite-dimensional Jordan superalgebras over an algebraically closed field of characteristic $p \neq 2$. As a consequence, a criterion for simple unital superalgebras of Jordan brackets to be special is obtained.
\end{abstract}

\section{INTRODUCTION}

The notion of a derivation of an algebra has been generalized by many mathematicians in various directions. In particular, in [1 one can find the definition of a $\delta$-derivation of an algebra. We recall that for fixed $\delta \in F$, a $\delta$-derivation of an $F$-algebra $A$ is a linear map $\phi$ satisfying the condition

$$
\phi(x y)=\delta(\phi(x) y+x \phi(y))
$$

for any $x, y \in A$. In [1], the $\frac{1}{2}$-derivations were described for an arbitrary prime Lie $F$-algebra $A\left(\frac{1}{6} \in F\right)$ with a nondegenerate symmetric invariant bilinear form. Namely, it was proved that a linear map $\phi: A \rightarrow A$ is a $\frac{1}{2}$-derivation if and only if $\phi \in \Gamma(A)$, where $\Gamma(A)$ is the centroid of $A$. This implies that if $A$ is a central simple Lie algebra over a field of characteristic $p \neq 2,3$ with a nondegenerate invariant bilinear form, then any $\frac{1}{2}$-derivation $\phi$ has the form $\phi(x)=\alpha x$ for some $\alpha \in F$.

In 2. Filippov proved that any prime Lie $\Phi$-algebra admits no nonzero $\delta$-variations if $\delta \neq-1,0, \frac{1}{2}, 1$. Also in [2], it was shown that any prime Lie $\Phi$-algebra $A\left(\frac{1}{6} \in \Phi\right)$ with a nonzero antiderivation is a three-dimensional central simple algebra over the field of fractions of the center $Z_{R}(A)$ for its algebra of right multiplications $R(A)$. In the same paper, a nontrivial $\frac{1}{2}$-derivation was constructed for the Witt algebra $W_{1}$, i.e., a $\frac{1}{2}$-derivation that is not an element of the centroid of $W_{1}$.

The paper [3] contains a description for the $\delta$-derivations of the prime alternative and non-Lie Maltsev $\Phi$-algebras with some restrictions on the operator ring $\Phi$. It was shown that the algebras in these classes have no nonzero $\delta$-derivations if $\delta \neq 0, \frac{1}{2}, 1$. The results of Filippov were partially generalized by Luks and Leger in [9. Those authors considered quasiderivations of Lie algebras, i.e., linear maps $f$ for which there exists a linear map $f^{\prime}$ related to $f$ by the formula $f^{\prime}(x y)=f(x) y+x f(y)$. They proved that

2010 Mathematics Subject Classification. Primary 17A70.

Key words and phrases. $\delta$-superdifferentiation, $\delta$-differentiation, Jordan superalgebra, Cantor double.

Supported by the Analytic Departmental Special Program "Development of the Scientific potential of Higher School" of the Federal Educational Agency (project 2.1.1.419), by RFBR grants nos. 0901-00157-A, 11-01-00938-A, by RF President Grant council for support of young scientists and leading scientific schools (project NSh-3669.2010.1), by Special Federal program "Scientific and Pedagogical staff of innovative Russia" for 2009-2013 (state contracts nos. 02.740.11.0429, 02.740.11.5191, 14.740.11.0346), by integrational project of SD RAS no. 97, and by Lavrent'ev grant for young scientists' collectives by SD RAS, Decision of the Presidium of SD RAS no. 43 of 04.02.2010. 
the space of quasiderivations of a simple finite-dimensional Lie algebra $A$ of rank greater than 1 coincides with the direct sum of the space of derivations and the centroid of $A$.

In [4, a description of $\delta$-derivations was given for the simple finite-dimensional Jordan superalgebras over an algebraically closed field of characteristic zero and for the semisimple finite-dimensional Jordan algebras over an algebraically closed field of characteristic different from 2 . Later, in [5, the $\delta$-derivations were described for the classical Lie superalgebras. The paper [6] was devoted to a description of the $\delta$-derivations of Cartan Lie superalgebras. In that paper, the $\delta$-superderivations were described for the simple finite-dimensional Lie superalgebras. Also in [6], the $\delta$-derivations of semisimple finite-dimensional Jordan algebras and $\delta$-superderivations of Jordan superalgebras over an algebraically closed field of characteristic zero were described. For algebras and superalgebras from the papers [4]-[6], the absence of nontrivial $\delta$-derivations and $\delta$-superderivations was proved. Later, the results of [5] were generalized by Zusmanovich in [8]. He described the $\delta$-derivations and $\delta$-superderivations of prime Lie superalgebras. He showed that a prime Lie superalgebra has no nontrivial $\delta$-derivations and $\delta$-superderivations if $\delta \neq-1,0, \frac{1}{2}, 1$. He proved that for a Lie superalgebra $A$ with zero center and a nondegenerate supersymmetric invariant bilinear form satisfying the condition $A=[A, A]$, the space of $\frac{1}{2}$-derivations ( $\frac{1}{2}$-superderivations) coincides with the centroid (supercentroid) of the superalgebra $A$. Also, Zusmanovich gave an affirmative answer to the question of Filippov in [2] concerning the existence of zero divisors in the ring of $\frac{1}{2}$-derivations of a prime Lie algebra. Subsequently, the $\delta$-superderivations of the generalized Kantor double constructed on a prime nonunital associative algebra were considered in [7].

In the present paper, we consider the $\delta$-derivations and $\delta$-superderivations in the case of simple superalgebras of Jordan brackets. We prove the absence of nontrivial $\delta$-derivations and $\delta$-superderivations of simple superalgebras of Jordan brackets, which are not superalgebras of vector type. A description is given for the $\delta$-derivations and $\delta$-superderivations of simple Jordan superalgebras of vector type. As a consequence, with the help of the classification of all simple finite-dimensional Jordan superalgebras, as given in [13, we obtain a description of the $\delta$-derivations and $\delta$-superderivations of the simple unital finite-dimensional Jordan superalgebras over an algebraically closed field of characteristic $p \neq 2$.

\section{$\S 1$. The MAIN FACTS AND DEFInitions}

Let $F$ be a field of characteristic $p \neq 2$. An algebra $A$ over the field $F$ is said to be Jordan if it satisfies the identities

$$
x y=y x, \quad\left(x^{2} y\right) x=x^{2}(y x) .
$$

Let $G$ be a Grassmann algebra over $F$, given by generators $1, \xi_{1}, \ldots, \xi_{n}, \ldots$ and the defining relations $\xi_{i}^{2}=0, \xi_{i} \xi_{j}=-\xi_{j} \xi_{i}$. The unity 1 and the products $\xi_{i_{1}} \xi_{i_{2}} \cdots \xi_{i_{k}}$, $i_{1}<i_{2}<\cdots<i_{k}$, form a basis of the algebra $G$ over $F$. Denote by $G_{0}$ and $G_{1}$ the subspaces generated by products of even and odd length, respectively; then $G$ is a direct sum of these subspaces: $G=G_{0} \oplus G_{1}$; moreover, we have the inclusions $G_{i} G_{j} \subseteq G_{i+j}$ $(\bmod 2), i, j=0,1$. In other words, $G$ is a $\mathbb{Z}_{2}$-graded algebra (or a superalgebra) over $F$.

Now, let $A=A_{0} \oplus A_{1}$ be an arbitrary superalgebra over $F$. Consider the tensor product $G \otimes A$ of $F$-algebras. Its subalgebra

$$
G(A)=G_{0} \otimes A_{0}+G_{1} \otimes A_{1}
$$

is called the Grassmann envelope of the superalgebra $A$.

Let $\Omega$ be a variety of algebras over $F$. A superalgebra $A=A_{0} \oplus A_{1}$ is called an $\Omega$-superalgebra if its Grassmann envelope $G(A)$ is an algebra in $\Omega$. 
In particular, $J=J_{0} \oplus J_{1}$ is a Jordan superalgebra if its Grassmann envelope $G(J)$ is a Jordan algebra. In what follows, for a homogeneous element $x$ of a superalgebra $J=J_{0} \oplus J_{1}$ we put $p(x)=i$ if $x \in J_{i}$. We denote the even part $J_{0}$ of a Jordan superalgebra by $A$ and the odd part $J_{1}$ by $M$.

A classification of the simple finite-dimensional Jordan superalgebras over an algebraically closed field of characteristic zero was given in [10, 11. In [12, 13, all simple finite-dimensional Jordan superalgebras over an algebraically closed field of arbitrary characteristic different from 2 were described.

We give several examples of Jordan superalgebras.

1.1. The Kantor double [10. Let $\Gamma=\Gamma_{0} \oplus \Gamma_{1}$ be an associative supercommutative superalgebra with unity 1 , and let $\{\}:, \Gamma \times \Gamma \rightarrow \Gamma$ be a superskewsymmetric bilinear map, which we shall call a bracket. Starting with the superalgebra $\Gamma$ and the bracket $\{$,$\} , one can construct a superalgebra J(\Gamma,\{\}$,$) . Consider the following direct sum of$ spaces: $J(\Gamma,\{\})=,\Gamma \oplus \Gamma x$, where $\Gamma x$ is an isomorphic copy of the space $\Gamma$. Let $a$ and $b$ be homogeneous elements in $\Gamma$. Then multiplication $\cdot$ on $J(\Gamma,\{\}$,$) is defined by the$ relations

$$
a \cdot b=a b, \quad a \cdot b x=(a b) x, \quad a x \cdot b=(-1)^{p(b)}(a b) x, \quad a x \cdot b x=(-1)^{p(b)}\{a, b\} .
$$

We set $A=\Gamma_{0} \oplus \Gamma_{1} x$ and $M=\Gamma_{1} \oplus \Gamma_{0} x$. Then $J(\Gamma,\{\})=,A \oplus M$ is a $\mathbb{Z}_{2}$-graded algebra.

A bracket $\{$,$\} is said to be Jordan if the superalgebra J(\Gamma,\{\}$,$) is a Jordan superal-$ gebra. It is well known [14] that, for homogeneous elements, the Jordan bracket satisfies the relations

$$
\begin{aligned}
\{a, b c\}= & \{a, b\} c+(-1)^{p(a) p(b)} b\{a, c\}-\{a, 1\} b c, \\
\{a,\{b, c\}\}= & \{\{a, b\}, c\}+(-1)^{p(a) p(b)}\{b,\{a, c\}\}+\{a, 1\}\{b, c\} \\
& +(-1)^{p(a)(p(b)+p(c))}\{b, 1\}\{c, a\}+(-1)^{p(c)(p(a)+p(b))}\{c, 1\}\{a, b\} .
\end{aligned}
$$

Since the superalgebra $J(\Gamma,\{\}$,$) is Jordan, we see that D: a \rightarrow\{a, 1\}$ is a derivation of the superalgebra $\Gamma$.

If $D$ is the zero derivation, then $\{$,$\} is a Poisson bracket, i.e.,$

$$
\{a, b c\}=\{a, b\} c+(-1)^{p(a) p(b)} b\{a, c\},
$$

and $\Gamma$ is a Lie superalgebra with respect to the operation $\{$,$\} . An arbitrary Poisson$ bracket is a Jordan bracket (see [15]).

It is well known 14 that the Jordan superalgebra $J=\Gamma \oplus \Gamma x$ obtained with the help of the Kantor doubling process is simple if and only if $\Gamma$ has no nonzero ideals $B$ satisfying $\{\Gamma, B\} \subseteq B$.

1.2. A superalgebra of vector type $J(\Gamma, D)$. Let $\Gamma=\Gamma_{0} \oplus \Gamma_{1}$ be an associative supercommutative superalgebra with a nonzero even derivation $D$. We define a bracket $\{$,$\} on \Gamma$ by setting

$$
\{a, b\}=D(a) b-a D(b) .
$$

Then the bracket $\{$,$\} is Jordan. We denote by J(\Gamma,\{\}$,$) the resulting superalgebra$ $J(\Gamma, D)$. Multiplication "." in $J(\Gamma, D)$ is defined by

$$
\begin{aligned}
a \cdot b=a b, \quad a \cdot b x & =(a b) x, \quad a x \cdot b=(-1)^{p(b)}(a b) x, \\
a x \cdot b x & =(-1)^{p(b)}(D(a) b-a D(b)),
\end{aligned}
$$

where $a$ and $b$ are homogeneous elements in $\Gamma$ and $a b$ is the product in $\Gamma$. The superalgebra $J(\Gamma, D)$ is called a superalgebra of vector type. If the superalgebra $J(\Gamma, D)$ is simple, then $\Gamma_{1}=0$ (see [14]). 
1.3. The Cheng-Kac superalgebra $C K(Z, d)$ [16]. Let $Z$ be an arbitrary unital associative commutative algebra with a nonzero derivation $d: Z \rightarrow Z$. Consider two free $Z$-modules of rank 4:

$$
A=Z+\sum_{i=1}^{3} w_{i} Z, \quad M=x Z+\sum_{i=1}^{3} x_{i} Z .
$$

Multiplication on $A$ will be $Z$-linear, $w_{i} w_{j}=0, i \neq j, w_{i}^{2}=-1$. We define

$$
x_{i \times i}=0, \quad x_{1 \times 2}=-x_{2 \times 1}=x_{3}, \quad x_{1 \times 3}=-x_{3 \times 1}=x_{2}, \quad x_{2 \times 3}=-x_{3 \times 2}=x_{1} .
$$

Multiplication $A \times M \rightarrow M$ is defined by the relations

$$
\begin{aligned}
(x f) g & =x(f g), & & \left(x_{i} f\right) g=x_{i}(f g), \\
(x f)\left(w_{j} g\right) & =x_{j}(f d(g)), & & \left(x_{i} f\right)\left(w_{j} g\right)=x_{i \times j}(f g) .
\end{aligned}
$$

Multiplication $M \times M \rightarrow A$ is given in accordance with the rules

$$
\begin{aligned}
(x f)(x g) & =d(f) g-f d(g), & & (x f)\left(x_{j} g\right)=-w_{j}(f g), \\
\left(x_{i} f\right)(x g) & =w_{i}(f g), & & \left(x_{i} f\right)\left(x_{j} g\right)=0 .
\end{aligned}
$$

We also need the definition of a certain superalgebra $B(n, m)$. Let $F$ be an algebraically closed field of characteristic $p>2$. Let $B(m)=F\left[a_{1}, \ldots, a_{m} \mid a_{i}^{p}=0\right]$ be the algebra of truncated polynomials in $m$ even variables. Let $G(n)$ be the Grassmann superalgebra with generators $1, \xi_{1}, \ldots, \xi_{n}$. Then $B(m, n)=B(m) \otimes G(n)$ is an associative supercommutative superalgebra.

The main result concerning classification of simple finite-dimensional unital Jordan superalgebras over algebraically closed fields of characteristic $p>2$ was obtained in the paper [13] by Martinez and Zelmanov.

Theorem 1. Let $J=J_{0}+J_{1}$ be a finite-dimensional simple unital Jordan superalgebra over an algebraically closed field of characteristic $p>2$, where $J_{0}$ is not a semisimple algebra. Then:

1) either there exist integers $m$ and $n$ and a Jordan bracket $\{$,$\} on B(m, n)$ such that $J=J(B(m, n),\{\}$,$) ,$

2) or $J$ is isomorphic to the Cheng-Kac Jordan superalgebra $C K(B(m), d)$, which is defined by the derivation $d: B(m) \rightarrow B(m)$.

As has been mentioned above, for a fixed element $\delta$ of the ground field, by a $\delta$-derivation of a superalgebra $A$ we mean a linear map $\phi: A \rightarrow A$ such that

$$
\phi(x y)=\delta(\phi(x) y+x \phi(y))
$$

for all $x, y \in A$.

The centroid $\Gamma(A)$ of a superalgebra $A$ is the set of all linear maps $\chi: A \rightarrow A$ such that

for all $x, y \in A$.

$$
\chi(x y)=\chi(x) y=x \chi(y)
$$

Note that the 1-derivation is the usual derivation and the 0-derivation is an arbitrary endomorphism $\phi$ of the algebra $A$ such that $\phi\left(A^{2}\right)=0$. It is clear that any element of the centroid of an algebra is a $\frac{1}{2}$-derivation.

A nonzero $\delta$-derivation $\phi$ is said to be nontrivial if $\delta \neq 0,1$ and $\phi \notin \Gamma(A)$.

By a superspace we mean a $\mathbb{Z}_{2}$-graded space. A homogeneous element $\psi$ of the superspace of endomorphisms $A \rightarrow A$ is called a superderivation if

$$
\psi(x y)=\psi(x) y+(-1)^{p(x) p(\psi)} x \psi(y) .
$$


For a fixed element $\delta \in F$, we define the notion of a $\delta$-superderivation of the superalgebra $A=A_{0}+A_{1}$. A homogeneous linear map $\phi: A \rightarrow A$ is called a $\delta$-superderivation if for homogeneous $x, y \in A$ we have

$$
\phi(x y)=\delta\left(\phi(x) y+(-1)^{p(x) p(\phi)} x \phi(y)\right) .
$$

Consider a Lie superalgebra $A=A_{0}+A_{1}$ and fix an element $x \in A_{i}$. Then $R_{x}: y \rightarrow$ $x y$ is an odd superderivation of the superalgebra $A$ and its parity $p\left(R_{x}\right)$ is equal to $i$.

The supercentroid $\Gamma_{s}(A)$ of a superalgebra $A$ is the set of all homogeneous linear maps $\chi: A \rightarrow A$ such that for arbitrary homogeneous elements $a$ and $b$, we have

$$
\chi(a b)=\chi(a) b=(-1)^{p(a) p(\chi)} a \chi(b) .
$$

Note that a 1-superderivation is a usual superderivation; a 0 -superderivation is an arbitrary endomorphism $\phi$ of the superalgebra $A$ such that $\phi\left(A^{2}\right)=0$.

A nonzero $\delta$-superderivation $\phi$ is said to be nontrivial if $\delta \neq 0,1$ and $\phi \notin \Gamma_{s}(A)$.

In accordance with [4, Theorem 2.1] (which is easily generalized to the case of $\delta$-superderivations), for a unital superalgebra $A$, a map $\phi$ can be a nontrivial $\delta$-derivation or $\delta$-superderivation only for $\delta=\frac{1}{2}$. It is easily seen that, in this case, $\phi(x)=\phi(1) x$ for arbitrary $x \in A$.

\section{$\S 2 . \delta$-DERIVATIONS AND $\delta$-SUPERDERIVATIONS OF SIMPLE SUPERALGEBRAS OF JORDAN BRACKETS}

In the present section, we consider $\delta$-derivations and $\delta$-superderivations of a simple unital Jordan superalgebra $J=J(\Gamma,\{\}$,$) . We assume that the characteristic of the field$ $F$ is different from 2 .

Lemma 2. Let $J=J(\Gamma,\{\}$,$) be a simple unital Jordan superalgebra. Then \Gamma=\Gamma\{\Gamma, \Gamma\}$. In particular, if $z \in \Gamma_{0} \cup \Gamma_{1} \backslash\{0\}$, then $z\{\Gamma, \Gamma\} \neq 0$.

Proof. Consider $I=\Gamma\{\Gamma, \Gamma\}$. It is clear that $I$ is an ideal in $\Gamma(I \triangleleft \Gamma)$. By (1),

$$
\{\Gamma, I\}=\{\Gamma, \Gamma\{\Gamma, \Gamma\}\} \subseteq\{\{\Gamma, \Gamma\},\{\Gamma, \Gamma\}\}+\Gamma\{\Gamma,\{\Gamma, \Gamma\}\}+\{\Gamma, 1\} \Gamma\{\Gamma, \Gamma\} \subseteq \Gamma\{\Gamma, \Gamma\}=I .
$$

By [14, the Jordan superalgebra $J(\Gamma,\{\}$,$) is simple if \Gamma$ contains no nonzero ideals $I$ satisfying the condition $\{\Gamma, I\} \subseteq I$. If $\{\Gamma, \Gamma\}=0$, then $\Gamma x \triangleleft J$. Consequently, $\Gamma x=J$ and $\Gamma_{0}=0$. Hence, $\{\Gamma, \Gamma\} \neq 0$, and since $\Gamma$ is unital, we have $\Gamma=\Gamma\{\Gamma, \Gamma\}$. Now if $z\{\Gamma, \Gamma\}=0$, then $z \Gamma=z \Gamma\{\Gamma, \Gamma\}=0$. Since $\Gamma$ is unital, we obtain $z=0$. The lemma is proved.

Lemma 3. Let $J=J(\Gamma,\{\}$,$) be a simple unital Jordan superalgebra, and let \alpha \in J$. The map $\phi(z)=\alpha z$ is a $\frac{1}{2}$-derivation if and only if $\alpha \in \Gamma_{0}$ and $\{\alpha, b\}=D(\alpha) b-\alpha D(b)$ for any $b \in \Gamma$.

Proof. Let $\alpha=\alpha_{0}+\beta x+\gamma+\mu x$, where $\alpha_{0}, \mu \in \Gamma_{0}$ and $\beta, \gamma \in \Gamma_{1}$. Clearly, the maps $\phi_{1}(z)=(\gamma+\mu x) z$ and $\phi_{2}(z)=\left(\alpha_{0}+\beta x\right) z$ are also $\frac{1}{2}$-derivations of the superalgebra $J$. Moreover, $\phi_{1}(1)=(\gamma+\mu x)$ and $\phi_{2}(1)=\left(\alpha_{0}+\beta x\right)$. For this reason, for arbitrary $z$, $w \in J$ we have

$$
2 \phi_{i}(1)(z w)=\left(\phi_{i}(1) z\right) w+z\left(\phi_{i}(1) w\right) .
$$

Setting $i=1, z=x$, and $w=1$ in (3), we get

$$
2 \gamma x=\gamma x+x \gamma=0
$$

i.e., $\gamma=0$.

We prove that $\beta=\mu=0$. For this, we show that $\beta\{\Gamma, \Gamma\}=0$ and $\mu\{\Gamma, \Gamma\}=0$. 
Now in (3) we set $i=2, z=a$, and $w=b x$, obtaining

$$
2\{\beta, a b\}=\{\beta a, b\}+(-1)^{p(a)} a\{\beta, b\} .
$$

By (1), we have

$$
\begin{aligned}
2\{\beta, a b\}=-(-1)^{p(b)+p(a) p(b)} 2\{b, \beta\} a & -(-1)^{p(a) p(b)} \beta\{b, a\} \\
& +(-1)^{p(b)+p(b) p(a)} D(b) \beta a .
\end{aligned}
$$

Substituting $i=2, z=a x$, and $w=b$ in (3), we get

$$
2\{\beta, a b\}=\{\beta, a\} b-(-1)^{p(a)}\{a, \beta b\} .
$$

By (1), we have

$$
2\{\beta, a b\}=2\{\beta, a\} b-\beta\{a, b\}-\beta D(a) b .
$$

Substituting $i=2, z=a x$, and $w=b x$ in (3), we get

$$
2 \beta\{a, b\}=\{\beta, a\} b-(-1)^{p(a)} a\{\beta, b\} .
$$

Comparison of (4) and (5) yields

$$
\begin{aligned}
2 \beta\{a, b\} & =2\{\beta, a\} b-\beta D(a) b-(-1)^{p(b)+p(b) p(a)} D(b) \beta a+(-1)^{p(b)+p(b) p(a)} 2\{b, \beta\} a \\
& =2\{\beta, a\} b-\beta D(a) b-\beta a D(b)-(-1)^{p(a)} 2 a\{\beta, b\} \\
& =2\{\beta, a\} b-\beta D(a b)-(-1)^{p(a)} 2 a\{\beta, b\} .
\end{aligned}
$$

This relation and (6) imply that

$$
2 \beta\{a, b\}=\beta D(a b) .
$$

Putting $b=1$, we get $\beta D(a)=2 \beta D(a)$. Hence, $\beta D(a)=0$ and $\beta\{\Gamma, \Gamma\}=0$. Thus, $\beta=0$ by Lemma 2 .

Substituting $i=1, z=a, w=b x$ in (3), we obtain

$$
2\{\mu, a b\}=-\{\mu a, b\}+(-1)^{p(a)} a\{\mu, b\} .
$$

Choosing $i=1, z=a x, w=b$ in (3), we get

$$
2\{\mu, a b\}=\{\mu, a\} b+(-1)^{p(a)}\{a, \mu b\} .
$$

For $i=1, z=a x, w=b x$ in (3), we have

$$
2 \mu\{a, b\}=\{\mu, a\} b+(-1)^{p(a)} a\{\mu, b\} .
$$

For $a=b=1$, relation (10) implies that $D(\mu)=0$. Substituting $b=1$ in (8), we obtain

$$
2\{\mu, a\}=\{\mu a, 1\}=D(\mu a)=\mu D(a) .
$$

Taking $a=1$ in (9), we have

$$
2\{\mu, b\}=\{1, \mu b\}=-D(\mu b)=-\mu D(b) .
$$

Comparing the expressions obtained, we get $\{\mu, \Gamma\}=0$. Therefore, $\mu\{a, b\}=0$ by (10). Using Lemma 2, we see that $\mu=0$.

Thus, we have proved that $\phi(z)=\alpha z$, where $\alpha \in \Gamma_{0}$.

Putting $i=2, z=a x$, and $w=b x$ in (3), we obtain

$$
2 \alpha\{a, b\}=\{\alpha a, b\}+\{a, \alpha b\} .
$$

Note that identity (11) yields the relation $\{\alpha a, b\}+\{a, \alpha b\}=-(-1)^{p(b) p(a)}(\{b, \alpha\} a+\alpha\{b, a\}-D(b) \alpha a)+\{a, \alpha\} b+\alpha\{a, b\}-D(a) \alpha b$.

Hence, (11) shows that

$$
a\{b, \alpha\}-\{a, \alpha\} b=(a D(b)-D(a) b) \alpha .
$$


Consequently, for $b=1$,

$$
\{\alpha, a\}=D(\alpha) a-\alpha D(a) .
$$

It is easy to verify that, for any $\alpha \in \Gamma_{0}$ such that $\{\alpha, a\}=D(\alpha) a-\alpha D(a), \phi(z)=\alpha z$ is a $\frac{1}{2}$-derivation of the superalgebra $J$. The lemma is proved.

Thus, a $\delta$-derivation of a simple unital superalgebra $J=J(\Gamma,\{\}$,$) is an even \delta$-superderivation of the superalgebra $J=J(\Gamma,\{\}$,$) . For this reason, in the sequel we deal only$ with $\delta$-superderivations.

Remark 4. Let $J=J(\Gamma,\{\}$,$) be a simple unital Jordan superalgebra. The map \phi(z)=$ $\alpha z$ is an odd $\frac{1}{2}$-superderivation if and only if $\alpha \in \Gamma_{1}$ and

$$
\{\alpha, a\}=D(\alpha) a-\alpha D(a)
$$

for any $a \in \Gamma$.

Proof. This is proved by straightforward calculations similar to those in the proof of Lemma 3.

Corollary 5. If $J$ is a simple unital superalgebra of vector type, then the map $\phi(z)=\alpha z$ is a $\frac{1}{2}$-superderivation if and only if $\alpha \in \Gamma_{0}$. If $J$ is a superalgebra of the Poisson bracket, then the map $\phi(z)=\alpha z$ is a $\frac{1}{2}$-superderivation if and only if $\alpha \in \Gamma_{0} \cup \Gamma_{1}$ and $\{\alpha, \Gamma\}=0$.

Let $J=J(\Gamma, D)$ be a superalgebra of vector type. The map $\phi(z)=\alpha z$ with $\alpha \in \Gamma$ is the trivial $\frac{1}{2}$-superderivation if $\phi \in \Gamma_{s}(J)$, i.e., if

$$
\alpha((b x)(c x))=(-1)^{p(\alpha) p(b x)}(b x)(\alpha(c x)),
$$

which is equivalent to $D(\alpha) b c=0$. Consequently, $\phi$ is the trivial $\frac{1}{2}$-superderivation if $D(\alpha) \neq 0$.

Let $J=J(\Gamma,\{\}$,$) be a superalgebra of the Poisson bracket, and let \phi(z)=\alpha z$ be a $\frac{1}{2}$-superderivation of $J$. By Remark 4, we have

$$
\begin{aligned}
(a x)(\alpha(b x)) & =(-1)^{p(b)+p(\alpha)}\{a, \alpha b\}=(-1)^{p(b)+p(\alpha)}\left(\{a, \alpha\} b+(-1)^{p(\alpha) p(a)} \alpha\{a, b\}\right) \\
& =(-1)^{p(b)+p(\alpha)+p(a) p(\alpha)} \alpha\{a, b\}=(-1)^{p(\alpha)(p(a)+1)} \alpha((a x)(b x)) .
\end{aligned}
$$

This easily implies that $\phi$ is the trivial $\frac{1}{2}$-superderivation.

We set

$$
\Phi=\left\{\alpha \in \Gamma_{0} \cup \Gamma_{1} \mid\{\alpha, a\}=D(\alpha) a-\alpha D(a), a \in \Gamma\right\} .
$$

Lemma 6. Suppose $J=J(\Gamma,\{\}$,$) is a simple Jordan superalgebra, \alpha \in \Phi \backslash\{0\}$, and $D(\alpha)=0$. Then $\alpha$ is invertible in $\Gamma$ and $\alpha \in \Gamma_{0}$. In particular, if $J(\Gamma,\{\}$,$) is a$ superalgebra of the Poisson bracket, then $\alpha$ is invertible in $\Gamma$ and $\alpha \in \Gamma_{0}$.

Proof. Consider $I=\alpha \Gamma$. It is clear that $I \triangleleft \Gamma$. By the definition of $\Phi$, we have

$$
\{I, \Gamma\}=\{\alpha \Gamma, \Gamma\} \subseteq\{\Gamma, \alpha\} \Gamma+\alpha\{\Gamma, \Gamma\}+D(\Gamma) \alpha \Gamma \subseteq \alpha \Gamma=I .
$$

Using [14, we conclude that $I=\Gamma$. Since $\Gamma$ is unital, it follows that $\alpha$ is invertible. The lemma is proved.

Lemma 7. Let $J=J(\Gamma,\{\}$,$) be a Jordan superalgebra. Then \Phi$ is closed with respect to the derivation $D$, i.e., $D(\Phi) \subseteq \Phi$. In particular, $D^{k}(\Phi) \subseteq \Phi, k>0$. 
Proof. Using (2), we obtain

$$
\begin{aligned}
\{D(b), c\} & +\{b, D(c)\}=-(-1)^{p(b) p(c)}\{c,\{b, 1\}\}+\{b,\{c, 1\}\} \\
= & -(-1)^{p(b) p(c)}\{\{c, b\}, 1\}-(-1)^{p(b) p(c)}(-1)^{p(c) p(b)}\{b,\{c, 1\}\} \\
& -(-1)^{p(b) p(c)}\{c, 1\}\{b, 1\}-(-1)^{p(b) p(c)}(-1)^{p(c) p(b)}\{b, 1\}\{1, c\}+\{\{b, c\}, 1\} \\
& +(-1)^{p(b) p(c)}\{c,\{b, 1\}\}+\{b, 1\}\{c, 1\}+(-1)^{p(b) p(c)}\{c, 1\}\{1, b\} \\
= & 2 D(\{b, c\})-\{D(b), c\}-\{b, D(c)\} .
\end{aligned}
$$

Consequently,

$$
D(\{b, c\})=\{D(b), c\}+\{b, D(c)\} .
$$

Therefore,

$$
\begin{aligned}
\{D(\alpha), a\} & =D(\{\alpha, a\})-\{\alpha, D(a)\} \\
& =D(D(\alpha)) a+D(\alpha) D(a)-D(\alpha) D(a)-\alpha D(D(a))-D(\alpha) D(a)+\alpha D(D(a)) \\
& =D(D(\alpha)) a-D(\alpha) D(a) .
\end{aligned}
$$

This completes the proof of the lemma.

Lemma 8. Let $J=J(\Gamma,\{\}$,$) be a Jordan superalgebra. Then for arbitrary b, c \in J$ and $\alpha \in \Phi$ we have

$$
D^{k}(\alpha)\{b, c\}=D^{k}(\alpha)(D(b) c-b D(c)) .
$$

Proof. From the definition of $\Phi$ and (12), it follows that

$$
\{\alpha,\{b, c\}\}=D(\alpha)\{b, c\}-\alpha D(\{b, c\})=D(\alpha)\{b, c\}-\alpha\{D(b), c\}-\alpha\{b, D(c)\} .
$$

Now we use relations (11), (2) and Lemmas 3 and 7 to write

$$
\begin{aligned}
\{\alpha,\{b, c\}\}=\{\{ & \alpha, b\}, c\}+(-1)^{p(\alpha) p(b)}\{b,\{\alpha, c\}\}+D(\alpha)\{b, c\} \\
& +(-1)^{p(\alpha)(p(b)+p(c))} D(b)\{c, \alpha\}+(-1)^{p(c)(p(\alpha)+p(b))} D(c)\{\alpha, b\} \\
=\{ & D(\alpha) b, c\}-\{\alpha D(b), c\}+(-1)^{p(\alpha) p(b)}\{b, D(\alpha) c\}-(-1)^{p(\alpha) p(b)}\{b, \alpha D(c)\} \\
& +D(\alpha)\{b, c\}-(-1)^{p(\alpha) p(b)} D(b)(D(\alpha) c-\alpha D(c)) \\
& +(-1)^{p(c)(p(\alpha)+p(b))} D(c)(D(\alpha) b-\alpha D(b)) \\
=- & -1)^{p(c)(p(\alpha)+p(b))}\{c, D(\alpha) b\}+(-1)^{p(c)(p(b)+p(\alpha))}\{c, \alpha D(b)\} \\
& +(-1)^{p(\alpha) p(b)}\{b, D(\alpha) c\}-(-1)^{p(\alpha) p(b)}\{b, \alpha D(c)\}+D(\alpha)\{b, c\} \\
& -(-1)^{p(\alpha) p(b)} D(b) D(\alpha) c+(-1)^{p(\alpha) p(b)} D(b) \alpha D(c) \\
& +(-1)^{p(c)(p(\alpha)+p(b))} D(c) D(\alpha) b-(-1)^{p(c)(p(\alpha)+p(b))} D(c) \alpha D(b) \\
= & (-1)^{p(c)(p(b)+p(\alpha))}\{c, D(\alpha)\} b-(-1)^{p(b) p(c)} D(\alpha)\{c, b\} \\
& +(-1)^{p(c)(p(\alpha)+p(b))} D(c) D(\alpha) b \\
& +(-1)^{p(c)(p(b)+p(\alpha))}\{c, \alpha\} D(b)+(-1)^{p(b) p(c)} \alpha\{c, D(b)\} \\
& +(-1)^{p(c)(p(\alpha)+p(b))} D(c) \alpha D(b)+(-1)^{p(\alpha) p(b)}\{b, D(\alpha)\} c+D(\alpha)\{b, c\} \\
& -(-1)^{p(\alpha) p(b)} D(b) D(\alpha) c-(-1)^{p(\alpha) p(b)}\{b, \alpha\} D(c)-\alpha\{b, D(c)\} \\
& +(-1)^{p(\alpha) p(b)} D(b) \alpha D(c)+D(\alpha)\{b, c\}-(-1)^{p(\alpha) p(b)} D(b) D(\alpha) c \\
& +(-1)^{p(\alpha) p(b)} D(b) \alpha D(c)+(-1)^{p(c)(p(\alpha)+p(b))} D(c) D(\alpha) b \\
& -(-1)^{p(c)(p(\alpha)+p(b))} D(c) \alpha D(b)
\end{aligned}
$$




$$
\begin{aligned}
=( & -1)^{p(b) p(c)} D(D(\alpha)) c b-(-1)^{p(c) p(b)} D(\alpha) D(c) b \\
& +D(\alpha)\{b, c\}+(-1)^{p(c)(p(\alpha)+p(b))} D(c) D(\alpha) b \\
& -(-1)^{p(b) p(c)} D(\alpha) c D(b)+(-1)^{p(b) p(c)} \alpha D(c) D(b)-\alpha\{D(b), c\} \\
& +(-1)^{p(c)(p(\alpha)+p(b))} D(c) \alpha D(b)-D(D(\alpha)) b c+D(\alpha) D(b) c+D(\alpha)\{b, c\} \\
& -(-1)^{p(\alpha) p(b)} D(b) D(\alpha) c+D(\alpha) b D(c)-\alpha D(b) D(c)-\alpha\{b, D(c)\} \\
& +(-1)^{p(\alpha) p(b)} D(b) \alpha D(c)+D(\alpha)\{b, c\}-(-1)^{p(\alpha) p(b)} D(b) D(\alpha) c \\
& +(-1)^{p(\alpha) p(b)} D(b) \alpha D(c)+(-1)^{p(c)(p(\alpha)+p(b))} D(c) D(\alpha) b \\
& -(-1)^{p(c)(p(\alpha)+p(c))} D(c) \alpha D(b) \\
=3 D & (\alpha)\{b, c\}-\alpha\{D(b), c\}-\alpha\{b, D(c)\}-2 D(\alpha)(D(b) c-b D(c)) .
\end{aligned}
$$

Therefore,

$$
3 D(\alpha)\{b, c\}-\alpha\{D(b), c\}-\alpha\{b, D(c)\}-2 D(\alpha)(D(b) c-b D(c))=\{\alpha,\{b, c\}\} .
$$

Comparing (13) and (14), we obtain

$$
D(\alpha)\{b, c\}=D(\alpha)(D(b) c-b D(c)) .
$$

Lemma 7 allows us to generalize this relation as follows:

$$
D^{k}(\alpha)\{b, c\}=D^{k}(\alpha)(D(b) c-b D(c)) .
$$

The lemma is proved.

Lemma 9. Let $J=J(\Gamma,\{\}$,$) be a simple unital Jordan superalgebra, and let \alpha \in \Phi$. If $D(\alpha) \neq 0$, then $J$ is a superalgebra of vector type. In particular, if $J$ is not a superalgebra of vector type, then $D(\alpha)=0$ and $\alpha$ is invertible in $\Gamma$.

Proof. We set $I=\Gamma D(\alpha)+\Gamma D^{2}(\alpha)+\cdots$. Note that $I \triangleleft \Gamma$. Using (1), the definition of $\Phi$, and Lemma 7 , it is easy to deduce that

$$
\begin{aligned}
\left\{\Gamma, \Gamma D^{k}(\alpha)\right\} & \subseteq\left\{\Gamma, D^{k}(\alpha)\right\} \Gamma+D^{k}(\alpha)\{\Gamma, \Gamma\}+D(\Gamma) D^{k}(\alpha) \Gamma \\
& \subseteq D(\Gamma) D^{k}(\alpha) \Gamma+\Gamma D^{k+1}(\alpha) \Gamma+D^{k}(\alpha)\{\Gamma, \Gamma\}+D(\Gamma) D^{k}(\alpha) \Gamma \\
& \subseteq \Gamma D^{k}(\alpha)+\Gamma D^{k+1}(\alpha),
\end{aligned}
$$

whence $\{\Gamma, I\} \subseteq I$. By [14], $\Gamma$ contains no nonzero ideals $I$ such that $\{\Gamma, I\} \subseteq I$. If $D(\alpha) \neq 0$, then $I=\Gamma$. Therefore, $1=\gamma_{1} D(\alpha)+\cdots+\gamma_{l} D^{l}(\alpha)$. Consequently, by Lemma 8 , for arbitrary $b, c \in \Gamma$ we get

$$
\begin{aligned}
\{b, c\} & =\left(\gamma_{1} D(\alpha)+\cdots+\gamma_{l} D^{l}(\alpha)\right)\{b, c\} \\
& =\gamma_{1} D(\alpha)(D(b) c-b D(c))+\cdots+\gamma_{l} D^{l}(\alpha)(D(b) c-b D(c))=D(b) c-b D(c) .
\end{aligned}
$$

Thus, $J$ is a superalgebra of vector type.

If $J$ is not a superalgebra of vector type, then the said above implies $D(\alpha)=0$. By Lemma $6, \alpha$ is an invertible element in $\Gamma$. The lemma is proved.

The above results are generalized in the following theorem.

Theorem 10. Let $J=J(\Gamma,\{\}$,$) be a simple unital superalgebra of a Jordan bracket over$ a field of characteristic different from 2. Then either $J$ has no nontrivial $\delta$-derivations and $\delta$-superderivations, or $J$ is a superalgebra of vector type. If $J$ is a superalgebra of vector type, then $\Gamma_{1}=0$ and the superalgebra $J$ has no nontrivial odd $\delta$-superderivations. For $\delta \neq \frac{1}{2}$, the superalgebra $J$ has no nontrivial $\delta$-derivations. The space of $\frac{1}{2}$-derivations coincides with $R^{*}(J)=\left\{R_{z} \mid z \in \Gamma_{0}\right\}$, and if $D(z) \neq 0$, then the map $R_{z}$ is a nontrivial $\frac{1}{2}$-derivation. 
Let $A=A_{0} \oplus A_{1}$ be an associative superalgebra. On the vector space $A$, we define a supersymmetric product $\circ$ by the rule

$$
a \circ b=\frac{1}{2}\left(a b+(-1)^{p(a) p(b)} b a\right) .
$$

We denote by $A^{(+)}$the algebra obtained. A Jordan superalgebra $B$ is said to be special if it is isomorphically embedded in the superalgebra $A^{(+)}$for a suitable associative superalgebra $A$.

Using Theorem 10 and the well-known fact that the unital Jordan superalgebras of vector type are special (see [14]), we obtain the following statement.

Corollary 11. If a simple unital superalgebra of a Jordan bracket $J=J(\Gamma,\{\}$,$) has a$ nontrivial $\delta$-derivation, then $J$ is special.

\section{$\S 3$. THE $\delta$-DERIVATIONS AND $\delta$-SUPERDERIVATIONS OF SIMPLE UNITAL FINITE-DIMENSIONAL JORDAN SUPERALGEBRAS}

Now we proceed to a description of the $\delta$-derivations and $\delta$-superderivations of simple unital finite-dimensional Jordan superalgebras over an algebraically closed field of characteristic $p \neq 2$. We recall that the $\delta$-derivations and $\delta$-superderivations of simple unital finite-dimensional Jordan superalgebras over an algebraically closed field of characteristic zero were described in [4, 6].

Recall that an algebra $A$ is said to be alternative if the identities

$$
(x, x, y)=0, \quad(x, y, y)=0
$$

are valid, where $(x, y, z)=(x y) z-x(y z)$ is the associator of elements $x, y, z \in A$. The algebra $O$ of octonions or Cayley numbers (see [17]) is a classical example of an alternative nonassociative algebra.

Now we give examples of simple nontrivial nonassociative alternative superalgebras of characteristic 3. Below $B$ denotes an alternative superalgebra over a field $F$, and $C$ and $M$ are the even and the odd part of $B$, respectively.

3.1. The superalgebra $B(1,2)$. Let $F$ be a field of characteristic 3 , and let $B(1,2)=$ $C+M$ be a supercommutative superalgebra over $F$ in which $C=F \cdot 1$ and $M=F \cdot x+F \cdot y$, where 1 is the unity of $B$ and $x y=-y x=1$. Note that the superalgebra $B(1,2)$ is precisely the simple Jordan superalgebra of the supersymmetric bilinear form $f(s, r)=s r$ on the odd vector space $M$.

3.2. The superalgebra $B(4,2)$. Let $F$ be a field of characteristic 3 , let $C=M_{2}(F)$ be the algebra of $(2 \times 2)$-matrices over $F$, and let $M=F \cdot m_{1}+F \cdot m_{2}$ be a two-dimensional irreducible Cayley bimodule over $C$, i.e., $C$ acts on $M$ in the following way:

$$
\begin{aligned}
e_{i j} \cdot m_{k} & =\delta_{i k} m_{j}, \quad i, j, k \in\{1,2\}, \\
m \cdot a & =\bar{a} \cdot m,
\end{aligned}
$$

where $a \in C, m \in M$, and $a \mapsto \bar{a}$ is the symplectic involution in $C=M_{2}(F)$. Odd multiplication on $M$ is defined by the relations

$$
m_{1}^{2}=-e_{21}, \quad m_{2}^{2}=e_{12}, \quad m_{1} m_{2}=e_{11}, \quad m_{2} m_{1}=-e_{22} .
$$

It is known (see [18]) that $B(1,2)$ and $B(4,2)$ are simple alternative superalgebras with the superinvolutions

$$
(a+m)^{*}=a-m \text { for } B(1,2), \quad(a+m)^{*}=\bar{a}-m \text { for } B(2,4) .
$$

An even linear transformation $*$ of a superalgebra $A$ is called a superinvolution if

$$
\left(a^{*}\right)^{*}=a,(a b)^{*}=(-1)^{p(a) p(b)} b^{*} a^{*}, \quad a, b \in A_{0} \cup A_{1} .
$$


In [18] it was shown that the superalgebras $B(1,2)$ and $B(4,2)$ give rise to the simple Jordan superalgebras $H_{3}(B(1,2))$ and $H_{3}(B(4,2))$.

Lemma 12. The superalgebras $H_{3}(B(1,2))$ and $H_{3}(B(2,4))$ have no nontrivial $\delta$-derivations and $\delta$-superderivations.

Proof. We denote by $e_{i j}$ the matrix units of the algebras $B(1,2)_{3}$ and $B(2,4)_{3}$. Let $\phi$ be a nontrivial $\delta$-derivation or $\delta$-superderivation. Clearly, $\delta=\frac{1}{2}$.

Let

$$
\phi\left(e_{i i}\right)=\sum_{j=1}^{3} \alpha_{j}^{i} e_{j j}+\sum_{k, l, k \neq l} x_{k l}^{i} e_{k l},
$$

where $x_{k l}^{i}=\overline{x_{l k}^{i}}$; then

$$
2 \phi\left(e_{i i}\right)=2 e_{i i} \circ \phi\left(e_{i i}\right)=2 \alpha_{i}^{i} e_{i i}+\sum_{k \neq i}\left(x_{i k}^{i} e_{i k}+x_{k i}^{i} e_{k i}\right) .
$$

Thus, $\phi\left(e_{i i}\right)=\alpha_{i}^{i} e_{i i}$. If $\beta \in F$ and

$$
\phi\left(\beta\left(e_{21}+e_{12}\right)\right)=\left(\begin{array}{ccc}
\gamma_{1} & x_{12} & x_{13} \\
\overline{x_{12}} & \gamma_{2} & x_{23} \\
\overline{x_{13}} & \overline{x_{23}} & \gamma_{3}
\end{array}\right),
$$

then

$$
\begin{gathered}
\left(\begin{array}{ccc}
0 & x_{12}+\frac{1}{2} \beta \alpha_{2}^{2} & 0 \\
\overline{x_{12}}+\frac{1}{2} \beta \alpha_{2}^{2} & 2 \gamma_{2} & x_{23} \\
0 & \overline{x_{23}} & 0
\end{array}\right)=\phi\left(\beta\left(e_{12}+e_{21}\right)\right) \circ e_{22}+\beta\left(e_{12}+e_{21}\right) \circ \phi\left(e_{22}\right) \\
=2 \phi\left(\left(\begin{array}{ccc}
0 & \beta & 0 \\
\beta & 0 & 0 \\
0 & 0 & 0
\end{array}\right) \circ e_{22}\right)=2 \phi\left(\left(\begin{array}{ccc}
0 & \beta & 0 \\
\beta & 0 & 0 \\
0 & 0 & 0
\end{array}\right) \circ e_{11}\right) \\
=\left(\begin{array}{ccc}
2 \gamma_{1} & x_{12}+\frac{1}{2} \beta \alpha_{1}^{1} & x_{13} \\
\overline{x_{12}}+\frac{1}{2} \beta \alpha_{1}^{1} & 0 & 0 \\
\overline{x_{13}} & 0 & 0
\end{array}\right) .
\end{gathered}
$$

This implies that $\alpha_{1}^{1}=\alpha_{2}^{2}=\alpha$. Similarly, we can show that $\alpha_{3}^{3}=\alpha$. Let $e=e_{11}+e_{22}+e_{33}$ be the unity of the superalgebra $H_{3}\left(B(1,2)\right.$ ) (or $H_{3}(B(2,4))$ ). Thus, in the case of a $\frac{1}{2}$-derivation and an even $\frac{1}{2}$-superderivation, we have $\phi(e)=\alpha e$, and in the case of an odd $\frac{1}{2}$-superderivation we have $\phi(e)=0$. This implies the triviality of $\phi$. The lemma is proved.

Lemma 13. Let $F$ be a field of characteristic $p>2$, and let $J=J(B(m, n),\{\}$,$) be a$ Jordan superalgebra that is not a superalgebra of vector type. Then $J$ has no nontrivial $\delta$-derivations and $\delta$-superderivations.

Proof. Lemma 3 shows that every $\delta$-derivation is an even $\delta$-superderivation. Let $\phi$ be a nontrivial $\delta$-superderivation. Clearly, $\delta=\frac{1}{2}$ and, by Lemma 3, $\phi(x)=\alpha x$, where $\alpha \in \Phi$. We see that $\alpha=\beta \cdot 1+r$, where $r$ is nilpotent and $\beta \in F$. Assume that $r \neq 0$. We may assume that $\alpha=r$, so that $\alpha$ is not invertible. By Lemma $6, D(\alpha) \neq 0$. Consequently, by Lemma $9, J$ is a superalgebra of vector type. This contradiction implies that $r=0$ and $\alpha \in F$. The lemma is proved.

Lemma 14. The superalgebra $C K(Z, d)$ has no nontrivial $\delta$-derivations and $\delta$-superderivations. 
Proof. Obviously, the even $\delta$-superderivations are $\delta$-derivations. Let $\phi_{0}$ be a nontrivial $\delta$-derivation and $\phi_{1}$ a nontrivial odd $\delta$-superderivation of the superalgebra $C K(Z, d)$. It is clear that $\delta=\frac{1}{2}$ and $\phi_{i}(x)=\phi_{i}(1) x$ for any $x \in C K(Z, d)$. We set

$$
\phi_{j}(1)=\alpha^{j}+\sum_{i=1}^{3} w_{i} \alpha_{i}^{j}+x \beta^{j}+\sum_{i=1}^{3} x_{i} \beta_{i}^{j} .
$$

Note that, since $\phi_{1}$ is homogeneous, we have $\alpha^{1}=\alpha_{i}^{1}=0$. We show that $\beta^{j}=\beta_{i}^{j}=$ $\alpha_{i}^{j}=0$.

It is easily seen that

$$
\begin{aligned}
0=\phi_{j}\left(x w_{k}\right)= & \frac{1}{2}(( \\
& \left.+(-1)^{j} x\left(\left(\alpha^{j}+\sum_{i=1}^{3} w_{i} \alpha_{i}^{j}+\beta^{j} x+\sum_{i=1}^{3} x_{i} w_{i} \alpha_{i}^{j}+\beta^{j} x+\sum_{i=1}^{3} x_{i} \beta_{i}^{j}\right) w_{k}\right)\right) \\
& =\frac{1}{2}\left(-\beta_{k}^{j}-(-1)^{j}\left(x \alpha_{k}^{j}-\sum_{i=1}^{3} w_{i \times k} \beta_{i}\right)\right) .
\end{aligned}
$$

This implies $\alpha_{i}^{j}=\beta_{i}^{j}=0$ and $\phi(1)=\alpha^{j}+\beta^{j} x$.

Now we have

$$
x_{i \times k} \alpha^{j}-w_{i \times k} \beta^{j}=\phi_{j}\left(x_{i} w_{k}\right)=\frac{1}{2}\left(\left(\phi(1) x_{i}\right) w_{k}+(-1)^{j} x_{i}\left(\phi(1) w_{k}\right)\right)=w_{i \times k} \alpha^{j},
$$

whence the required statement follows: $\phi_{0}(x)=\alpha^{0} x, \alpha^{0} \in Z$, and $\phi_{1}=0$, i.e., $\phi_{j}$ is trivial. The lemma is proved.

By the results of [12, 18, the simple unital finite-dimensional Jordan superalgebras with a semisimple even part over an algebraically closed field of characteristic $p>2$ are exhausted by the superalgebras $H_{3}(B(1,2))$ and $H_{3}(B(2,4))$, which are considered over fields of characteristic 3. By [13], the simple unital finite-dimensional Jordan algebras with a nonsemisimple even part over an algebraically closed field of characteristic $p>2$ are exhausted by the superalgebras $J=J(B(m, n),\{\}$,$) and C K(B(m), d)$. Thus, combining the above classification of simple unital Jordan superalgebras over an algebraically closed field of characteristic $p>2$, the results of [4, 6, Corollary 5, and Lemmas 12-14, we see that the following is true.

Theorem 15. Let $J$ be a simple unital finite-dimensional Jordan superalgebra over an algebraically closed field of characteristic $p \neq 2$. Then either $J$ has no nontrivial $\delta$ derivations and $\delta$-superderivations, or $J$ is a superalgebra of vector type over a field of characteristic $p>2$. If $J=J(B(m, n),\{\}$,$) is a superalgebra of vector type, then$ $n=0$ and the superalgebra $J$ has no nontrivial odd $\delta$-superderivations. For $\delta \neq \frac{1}{2}$, the superalgebra $J$ has no nontrivial $\delta$-derivations. The space of $\frac{1}{2}$-derivations coincides with $R^{*}(J)=\left\{R_{z} \mid z \in B(m)\right\}$, and for $D(z) \neq 0$ the map $R_{z}$ is a nontrivial $\frac{1}{2}$-derivation.

In conclusion, it should be noted that, while this paper was being published, the derivations of the Cheng-Kac superalgebras [19] and of the Kantor double of a simple unital Poisson superalgebra 20, were described. Also, a complete description was obtained for the $\delta$-(super)derivations of simple nonunital Jordan superalgebras over an algebraically closed field and, as a consequence, for the $\delta$-(super)derivations of semisimple finite-dimensional Jordan superalgebras over an algebraically closed field of characteristic different from 2 (see [21]). 


\section{REFERENCES}

[1] V. T. Filippov, On $\delta$-derivations of Lie algebras, Sibirsk. Mat. Zh. 39 (1998), no. 6, 1409-1422; English transl., Siberian Math. J. 39 (1998), no. 6, 1218-1230. MR.1672673 (2001a:17028)

[2] _ On $\delta$-derivations of prime Lie algebras, Sibirsk. Mat. Zh. 40 (1999), no. 1, 201-213; English transl., Siberian Math. J. 40 (1999), no. 1, 174-184. MR1686989(2000c:17033a)

[3] _ On $\delta$-derivations of prime alternative and Mal'tsev algebras, Algebra i Logika 39 (2000), no. 5, 618-625; English transl., Algebra and Logic 39 (2000), no. 5, 354-358. MR1805760 (2002e:17044)

[4] I. B. Kaygorodov, On $\delta$-derivations of simple finite-dimensional Jordan superalgebras, Algebra i Logika 46 (2007), no. 5, 585-605; English transl., Algebra and Logic 46 (2007), no. 5, 318-329. $\operatorname{MR} 2378632(2008 \mathrm{j}: 17060)$

[5] _ On $\delta$-derivations of classical Lie superalgebras, Sibirsk. Mat. Zh. 50 (2009), no. 3, 547-565; English transl., Siberian Math. J. 50 (2009), no. 3, 434-449. MR2555880 (2010k:17027)

[6] _ $\_$-superderivations of simple finite-dimensional Jordan and Lie superalgebras, Algebra $\mathrm{i}$ Logika 49 (2010), no. 2, 195-215; English transl., Algebra and Logic 49 (2010), no. 2, 130-144. MR2724805 (2012a:17063)

[7] - On the generalized Kantor's double, Vestnik Samar. Gos. Univ. 78 (2010), no. 4, 53-63. (Russian)

[8] P. Zusmanovich, On $\delta$-derivations of Lie algebras and superalgebras, J. Algebra 324 (2010), no. 12, 3470-3486. MR2735394 (2012a:17028)

[9] G. Leger and E. Luks, Generalized derivations of Lie algebras, J. Algebra 228 (2000), no. 1, 165203. MR.1760961 (2001e:17029)

[10] I. L. Kantor, Jordan and Lie superalgebras defined by Poisson algebras, Algebra and Analysis, Tomsk. Gos. Univ., Tomsk, 1989, pp. 55-80. (Russian) MR.1191172 (93j:17004)

[11] V. G. Kac, Classification of simple $\mathbb{Z}$-graded Lie superalgebras and simple Jordan superalgebras, Comm. Algebra 13 (1977), 1375-1400. MR0498755 (58:16806)

[12] M. L. Racine and E. I. Zelmanov, Simple Jordan superalgebras with semisimple even part, J. Algebra 270 (2003), no. 2, 374-444. MR2019625 (2005b:17063)

[13] C. Martinez and E. Zelmanov, Simple finite-dimensional Jordan superalgebras of prime characteristic, J. Algebra 236 (2001), no. 2, 575-629. MR.1813492(2002e:17042)

[14] D. King and K. McCrimmon, The Kantor construction of Jordan superalgebras, Comm. Algebra 20 (1992), no. 1, 109-126. MR.1145328 (92j:17032)

[15] I. L. Kantor, Connection between Poisson brackets and Jordan and Lie superalgebras, Lie Theory, Differential Equations and Representation Theory (Montreal, 1989), Univ. Montreal, Montreal, QC, 1990, pp. 213-225. MR.1121965 (92f:17040)

[16] S. J. Cheng and V. G. Kac, A new $N=6$ superconformal algebra, Comm. Math. Phys. 186 (1997), no. 1, 219-231. MR1462763 (99f:17029)

[17] K. A. Zhevlakov, A. M. Slin'ko, I. P. Shestakov, and A. I. Shirshov, Rings that are nearly associative, Nauka, Moscow, 1978; English transl., Pure Appl. Math., vol. 104, Acad. Press, Inc., New YorkLondon, 1982. MR0518614 (80h:17002), MR0668355 (83i:17001)

[18] I. P. Shestakov, Prime alternative superalgebras of arbitrary characteristic, Algebra i Logika 36 (1997), no. 6, 675-716; English transl., Algebra and Logic 36 (1997), no. 6, 389-412. MR.1657313 $(99 \mathrm{k}: 17006)$

[19] E. Barreiro, A. Elduque, and C. Martinez, Derivations of the Cheng-Kac Jordan superalgebras, J. Algebra 338 (2011), 144-156. MR2805185

[20] A. Retakh, Derivations of KKM doubles, Comm. Algebra 38 (2010), 3660-3670. MR2760682 (2011j:17053)

[21] I. B. Kaygorodov, On $\delta$-superderivations of semisimple finite-dimensional Jordan superalgebras, Mat. Zametki 91 (2012), no. 2, 200-213. (Russian); http://arxiv.org/abs/1106.2680v1.

Sobolev Institute of Mathematics, Sibir Branch of Russian Academy of Sciences, AcademiCian Koptyug Avenue 4, Novosibirsk 630090, Russia

E-mail address: vicnic@math.nsc.ru

Novosibirsk State University, Pirogov Street 2, Novosibirsk 630090, Russia

E-mail address: kib@math.nsc.ru 